\title{
Comparação do equilíbrio postural de idosos sedentários, praticantes de diferentes modalidades de exercícios e adultos jovens
}

http://dx.doi.org/10.11606/1807-5509201800020289

\author{
Raquel de Melo MARTINS* \\ Thiago Viana CAMATA* \\ Juliana Bayeux DASCAL* \\ Rubens Alexandre da SILVA JÚNIOR** \\ Inara MARQUES* \\ *Departamento de \\ Educação Física, \\ Universidade Estadual \\ de Londrina, Londrina, \\ PR, Brasil. \\ **Universidade Norte \\ do Paraná, Londrina, \\ PR, Brasil.
}

\section{Resumo}

0 objetivo deste estudo foi comparar o equilibrio postural de idosos praticantes de duas modalidades de exercícios físicos, idosos não praticantes e adultos jovens, na presença e ausência de informação visual. Participaram 51 indivíduos ( $n=31$ do sexo feminino e $n=20$ do sexo masculino), subdivididos em quatro grupos: idosos praticantes de karatê (GIKA, $n=11)$, idosos praticantes de ginástica generalizada (GIGG, $n=16$ ), idosos não praticantes de exercícios físicos (GINP, $n=12$ ), e um grupo controle de adultos jovens (GAJ, $n=12$ ). A tarefa constituiu em permanecer em apoio estático, posição semi tandem, sobre a plataforma de força por 40 segundos. Duas condições experimentais foram realizadas: com visão e sem visão. As variáveis de controle postural obtidas a partir do centro de pressão (Cop) foram: área de oscilação do Cop, velocidade e amplitude média de oscilação do Cop nas direções ântero-posterior e médio-lateral. Os resultados indicaram que os idosos praticantes de exercícios físicos GIKA e GIGG obtiveram desempenhos semelhantes, não diferenciando a modalidade praticada, tanto na condição com visão quanto sem visão, aproximando seu desempenho de adultos jovens, mais especificamente o grupo GIGG. Na condição sem visão, todos os grupos mostraram-se mais suscetiveis à instabilidade postural. 0 grupo GINP apresentou maior oscilação postural em todas as variáveis analisadas quando comparado aos outros grupos, apresentando maiores diferenças com o grupo GIGG e GAJ. Sendo assim, os grupos de idosos praticantes não se diferenciaram, se aproximando ao desempenho dos adultos jovens. Compreende-se que a prática de exercícios físicos tem papel importante e eficaz na manutenção do equilíbrio postural durante o envelhecimento e pode evitar possiveis quedas.

PalavRAS-Chave: Envelhecimento; Equilibrio postural; Exercícios físicos; Avaliação postural.

\section{Introdução}

Nas últimas décadas, têm-se observado um gradual aumento da população idosa no país ${ }^{1}$, juntamente a um maior interesse em pesquisas sobre os declínios significativos das capacidades físicas e funcionais de idosos durante o envelhecimento. Dentre as capacidades que sofrem declínio na velhice, destaca-se o equilíbrio postural ${ }^{2,3}$, sendo este relacionado a altos índices de quedas e fraturas, que podem levar à perda de mobilidade e à dependência física ${ }^{2,3}$.

Diferentes estudos têm mostrado que o exercício físico tem um papel importante na manutenção do equilibrio postural em idosos, atuando na prevençáo de perdas funcionais relacionadas ao envelhecimento ${ }^{4-10}$. Surge a questáo de como é o comportamento do equilíbrio postural em diferentes práticas motoras e modalidades esportivas e se estes apresentam respostas posturais distintas.

Alguns autores ${ }^{11-13}$ sugerem que o equilíbrio postural de praticantes de exercícios físicos pode ser atribuído pela experiência repetitiva em respostas motoras, assim como na melhora das funçóes proprioceptivas e de pistas visuais advindas do ambiente. A maioria desses estudos compara práticas esportivas em populaçóes mais jovens e são poucos estudos comparando diferentes práticas de exercícios físicos e esportivos e como ocorrem suas respostas comportamentais durante o envelhecimento ${ }^{6,8,14}$.

É comum a realização de delineamentos que utilizam práticas normalmente direcionadas a grupos de idosos, tais como hidroginástica ${ }^{15}$, caminhada ${ }^{16}$, 
treinamento com pesos ${ }^{14,17}$ tai chi chuan ${ }^{14,18}$ e yoga $^{19}$, os quais indicam uma melhora no desempenho do equilíbrio, por meio de uma menor oscilaçáo postural. Alguns estudos trazem os benefícios de treinamentos mais específicos para a prevenção de quedas, como meio de minimizar a instabilidade postural e melhorar as reaçóes posturais em diferentes contextos ${ }^{10,20}$.

Dessa maneira, modalidades como o Karatê e a Ginástica Generalizada, que abrangem diversificadas respostas comportamentais diante de situaçóes de equilíbrio estático e dinâmico, caracterizadas por perturbar constantemente o sistema de controle postural, ainda são pouco exploradas. Alguns autores ${ }^{21-24}$ discutem o efeito positivo do treinamento do Karatê, como prática de habilidades motoras que podem influenciar de maneira positiva, em respostas de ações motoras mais rápidas para o controle postural. Benefícios são a melhoria na aptidão, agilidade, concentração, autocontrole e mobilização, além de evidências de melhoras das funçôes cognitivas ${ }^{22,24}$. A ginástica generalizada é uma modalidade mais comum aos idosos, com exercícios que envolvam a coordenação motora, agilidade, equilíbrio, fortalecimento de membros inferiores e superiores ${ }^{24}$.

Sendo assim, umas das formas para comparar essas diferenças entre as práticas de karatecas e idosos praticantes de ginástica generalizada é por meio da manipulação dos canais sensoriais importantes para o equilíbrio ${ }^{25,26}$. Uma estratégia para verificar a influência sensorial é manipular tais estímulos recebidos e analisar as respostas motoras $^{26}$, como por exemplo, a oclusão do sistema visual $^{8,25}$. Nos exercícios físicos específicos, a prática pode modificar a influência do input visual em performances de equilíbrio ${ }^{27}$, beneficiando o participante. Além disso, compará-los a idosos não ativos e adultos jovens é uma forma de entender melhor os mecanismos dessas práticas e suas particularidades no equilíbrio postural.

Dessa forma, o objetivo deste estudo foi verificar se há diferenças de respostas na oscilação do equilíbrio postural entre duas modalidades de exercícios físicos em idosos praticantes de karatê e ginástica generalizada, comparados a idosos não praticantes e jovens adultos, na presença $\mathrm{e}$ ausência de informação visual. Nossa hipótese é que idosos praticantes de exercícios físicos irão apresentar melhor equilíbrio postural quando comparados aos idosos não praticantes em ambas as condições, e que os praticantes de karatê terão melhor equilíbrio postural do que os idosos praticantes de ginástica generalizada, devido às características das práticas.

\section{Método}

Participaram do estudo 51 indivíduos, voluntários e saudáveis, subdivididos em quatros grupos: praticantes de karatê (GIKA); 11 idosos (5 homens e 6 mulheres); idosos praticantes de

IMC - Índice de Massa Corporal, MEEM - Mini-exame do estado Mental; QBMI - Questionário de atividade física adaptado para idosos (Backer). Grupos: (GIKA) - Grupo de praticantes de Karatê; (GIGG) - Grupo de idosos praticantes de Ginástica

Generalizada; (GINP) - Grupo de Idosos não praticantes; (GAJ) - Grupo de Adultos Jovens. \# Diferença significativa entre GIGG e GAJ.

*Diferença significativa entre GINP e GAJ.

TABELA 1 - Característica dos participantes. e 9 mulheres); não praticantes de atividade física (GINP), 12 idosos (5 homens e 7 mulheres) e um grupo de adultos jovens (GAJ), 12 adultos jovens (4 homens e 8 mulheres). As características dos participantes estão representadas na TABELA 1.

\begin{tabular}{lcccc}
\hline \multicolumn{5}{c}{ Grupos } \\
\hline Gdade & GIKA $(\boldsymbol{n}=\mathbf{1 1})$ & GIGG $(\boldsymbol{n}=\mathbf{1 6})$ & GINP $(\boldsymbol{n}=\mathbf{1 2})$ & GAJ $(\boldsymbol{n}=\mathbf{1 2})$ \\
Estatura $(\mathrm{cm})$ & $69,83 \pm 3,88$ & $69,91 \pm 4,49$ & $70,91 \pm 4,44$ & $24,15 \pm 4,08$ \\
Massa corporal $(\mathrm{kg})$ & $161,50 \pm 8,85^{\#}$ & $155,84 \pm 7,73$ & $159,79 \pm 8,81^{*}$ & $170,19 \pm 9,25^{\# *}$ \\
IMC $\left(\mathrm{kg} / \mathrm{cm}^{2}\right)$ & $69,00 \pm 15,64$ & $67,86 \pm 12,28$ & $76,58 \pm 6,58$ & $66,12 \pm 11,93$ \\
Tempo de Prática (anos) & $26,23 \pm 4,23$ & $27,02 \pm 4,45^{*}$ & $30,12 \pm 2,03^{*}$ & $22,75 \pm 3,28^{\# *}$ \\
MEEM & $4,5(3-8)$ & $5(3-7)$ & --- & -- \\
QBMI & $26(24-8)$ & $23,5(22-26)$ & $23,5(21-27)$ & $-\cdots$ \\
\hline
\end{tabular}


Os critérios de inclusão foram:

Para o grupo de idosos praticantes de exercícios (GIKA e GIGG): praticar regularmente o programa de exercícios físicos por pelo menos duas vezes semanaisnos últimos seis meses e ter pontuação acima de 9 no Questionário de Baecke; ter idade entre 65 e 75 anos; estado cognitivo acima de 18, por meio do questionário Mini-mental; não apresentar limitaçóes físicas e/ou sensoriais que pudessem influenciar a realização das tarefas experimentais e náo praticar outros exercícios físicos e esportivos diferentes dos que já estavam praticando. Os grupos de não praticantes de exercícios físicos deveriam ter pontuação menor que 10 no questionário adaptado de Baecke e o de adultos jovens, não estarem engajados em práticas de exercícios físicos nos últimos seis meses.

Para tanto, foi aplicada uma anamnese para todos os participantes com o objetivo de conhecer os aspectos funcionais e físicos, por meio de histórico de quedas, tonturas e de medicaçôes. Os participantes foram previamente informados sobre os objetivos da pesquisa e os procedimentos adotados no estudo. Todos os participantes assinaram o termo de consentimento livre e esclarecido. Os procedimentos adotados na pesquisa obedeceram aos Critérios de Ética em Pesquisa com Seres Humanos, conforme resolução no 196/96, do Conselho Nacional de Saúde e aprovado pelo Comitê de Ética da Universidade local, sob o número 287/2011.

Os idosos Karatecas foram recrutados em uma academia de artes marciais do município de Londrina$\mathrm{PR}$, os mesmos alunos de uma turma composta, especificamente, por idosos. As aulas eram realizadas regularmente três vezes por semana e eram compostas de atividades envolvendo aquecimento articular e alongamentos como parte inicial das aulas, e de movimentos particulares ao Karatê, simulando golpes e movimentos dinâmicos, tanto com membros inferiores quanto superiores, como parte principal da aula. Já os idosos que faziam parte do grupo de ginástica geral, participavam de um programa dentro da Universidade local, com acompanhamento de professores e alunos do curso Educação Física, três vezes por semana, com duração de 60 minutos. Os exercícios envolviam atividades com equilíbrio, agilidade, coordenação motora e atividades esportivas, com manipulaçáo de bolas.

\section{Protocolo experimental}

O teste de equilíbrio postural consistiu na permanência dos participantes na posição ereta e estática, por 40 segundos, sobre uma plataforma de força, com os pés descalços e os braços estendidos ao lado do corpo. A base de suporte adotada durante o teste foi a semi-tandem e as condições experimentais delineadas para o estudo incluíram a condição com visão $(\mathrm{CV})$ e sem visão $(\mathrm{SV})$.

$\mathrm{Na}$ condição $\mathrm{CV}$, foi solicitado ao participante que olhasse diretamente para o centro de uma marcação preta, fixada na parede na altura dos olhos, a uma distância de 2 metros a partir do centro da plataforma. Na condição SV, os participantes deveriam permanecer na mesma posição solicitada na condição $\mathrm{CV}$, entretanto sua visão foi ocluída com uma venda preta.

As tarefas foram realizadas em três tentativas para cada condição experimental. Os participantes realizavam primeiro as três tentativas da condição determinada por meio de randomizaçáo, com intervalo de trinta segundos entre tentativas e para a outra condição, um intervalo de sessenta segundos, iniciando, dessa maneira, as coletas metade do grupo na condição CV e metade do grupo na condição SV.

As avaliaçóes ocorreram no Laboratório de Avaliação Funcional e Performance Motora Humana (LAFUP), da Universidade Norte do Paraná (UNOPAR). Os participantes foram avaliados individualmente pela equipe do laboratório, composta pela pesquisadora e mais dois assistentes. Antes de iniciarem o protocolo de coletas, a pressão arterial e a frequência cardíaca de repouso foram aferidas. Caso a frequência cardíaca apresentasse alteraçóes, com a pressão arterial sistólica ou diastólica alteradas, os idosos deveriam permanecer sentados, até a pressáo arterial se normalizar, para assim, realizarem os testes. Após os procedimentos iniciais, os participantes foram conduzidos à plataforma de força para a sessão de familiarização e depois foram submetidos às tarefas experimentais propostas. Para a segurança dos participantes, durante as condições experimentais, a equipe de apoio ficou sempre próxima à área da plataforma de força para evitar possíveis desequilíbrios.

Para a coleta do equilíbrio postural, utilizou-se de uma plataforma de força. O cálculo do Cop foi derivado de uma amostragem de $100 \mathrm{~Hz}$, todos os sinais de força registrados foram filtrados com um filtro de banda-passante baixo de 35-Hz e de segunda ordem Butterworth filter. Para aquisição e tratamento dos parâmetros de equilíbrio, foi utilizado o próprio software Bioanalysis da plataforma BIOMEC400, o qual é compilado com rotinas de computação MATLAB (The Mathworks, Natick, MA). 
Para avaliação do equilíbrio postural, as seguintes variáveis dependentes foram obtidas a partir do Cop:

1) Área de deslocamento do Cop $\left(\mathrm{cm}^{2}\right)$, calculada por meio do cálculo da área da elipse que engloba 95\% da amplitude total de deslocamento;

2) Velocidade média de deslocamento do Cop, nas direções AP e ML $(\mathrm{cm} / \mathrm{s})$;

3) Amplitude média de deslocamento do Cop, nas direçôes AP e ML (cm).

Após coletadas as variáveis do Cop, utilizou-se da média das três tentativas para as análises.

A validade e confiabilidade (com base no coeficiente de correlação infraclasses: ICC> 0,80 e erro médio padronizado: SEM $<1,30)$ para esses parâmetros calculados com esta plataforma de força e software, foram aceitos para adultos jovens e idosos, como relatado em estudos prévios ${ }^{28,29}$.

\section{Análise estatística}

Primeiramente, a normalidade dos dados foi avaliada por meio do teste de Shapiro Wilk, a homogeneidade das variâncias pelo teste de Levene, e a esfericidade por meio do teste de Mauchly. Para verificar a diferença entre os quatro grupos, foi utilizada uma análise de variância de dois fatores (ANOVA Two-way: Grupos x Condiçôes) com medidas repetidas no fator condições. Foi utilizado o post hoc de Tukey para localizar as diferenças entre os grupos. O pacote estatístico adotado foi o Statistica 7, com o nível de significância adotado $P \leq 0,05$.

\section{Resultados}

Os valores da área de oscilação postural do Cop, são apresentados na FIGURA 1.

Houve diferenças significativas entre os grupos $(\mathrm{F}(3,47=10,063, \mathrm{p}<0,001)$ e condiçōes $(\mathrm{F}(1,47)=91,495, \mathrm{p}<0,001)$. O teste post hoc apontou que o grupo GINP se diferenciou dos demais grupos, na condiçấo sem visão, com um maior deslocamento do Cop. Não houve interação entre grupos x condiçôes. $\mathrm{Na}$ análise intragrupos, todos os grupos apresentaram diferenças nas condiçóes experimentais com e sem visão, com uma maior oscilação $(\mathrm{p}<0,05)$.

$\mathrm{Na}$ FIGURA 2, são apresentados os valores de amplitude, na direção AP do Cop.

Houve diferença significativa entre os grupos $(\mathrm{F}(3,47)=9,6761, \mathrm{p}<0,01)$ e entre as condiçôes $(\mathrm{F}(1,47)=30,927, \mathrm{p}<0,01)$. O teste post hoc indicou que o grupo GINP apresentou diferenças em relação aos grupos GIGG e GAJ na condiçáo $\mathrm{SV}$, com o grupo GINP apresentando uma maior oscilação postural que estes grupos, nesta condição $(\mathrm{p}<0,05)$. Não houve interação grupos $x$ condiçôes. Na avaliaçáo intragrupos, os grupos GIKA e GINP apresentaram diferenças entre as condiçóes com visão $(\mathrm{CV})$ e sem visão (SV), com um maior deslocamento do Cop, na condição $S V(p<0,05)$.

Na FIGURA 3, sáo apresentados os valores de amplitude, na direção ML do Cop.

Pode-se observar que houve diferenças significativas entre os grupos $(\mathrm{F}(3,47)=8,45, \mathrm{p}<0,01)$ e entre condições $(F(1,47)=86,361, p<0,01)$. $O$ teste de

post hoc mostrou que, em comparação entre os grupos, o GINP foi diferente do GIGG e GAJ, com um maior deslocamento do Cop, na direçáo ML, para o grupo GINP, nas condiçóes CV e SV $(p<0,05)$. Não houve interação grupos x condiçóes. A comparação intragrupo mostrou que todos os grupos mostraram diferenças nas duas condiçôes experimentais $(\mathrm{CV}$ e $\mathrm{SV}, p<0,05)$, indicando uma maior amplitude de oscilação na condição sem visão.

$\mathrm{Na}$ FIGURA 4, são apresentados os valores médios de velocidade de oscilação, na direção AP. Houve diferença significativa entre o grupos $(\mathrm{F}(3,47)=8,7286, \mathrm{p}<0,01)$ e entre condições $(\mathrm{F}(1,47)=50,482, \mathrm{p}<0,01)$. O post hoc indicou diferenças para os grupos GINP e GAJ $(p<0,05)$, com GINP demonstrando maior oscilação do Cop na condição $\mathrm{CV}$. Nâo houve interação grupos $\mathrm{x}$ condiçôes. A comparação intragrupo mostrou que os grupos de idosos mostraram diferenças nas duas condições experimentais ( $\mathrm{CV}$ e $\mathrm{SV}, p<0,05)$, com uma maior velocidade de oscilaçáo na condição $S V$.

$\mathrm{Na}$ FIGURA 5, são apresentados os valores médios de velocidade de oscilação, na direção ML. Houve diferença significativa entre os grupos, $(\mathrm{F}(3,47)=10,019, \mathrm{p}<0,01)$ e entre as condiçóes $(\mathrm{F}(1,47)=75,734, \mathrm{p}<0,01)$. O teste post hoc apontou diferenças entre grupos GIGG e GINP e entre GAJ e GINP $(\mathrm{p}<0,05)$, com o grupo GINP apresentando uma maior velocidade de oscilação na direção ML. Não houve interação entre grupos $\mathrm{x}$ condiçōes. 
A comparação intragrupo mostrou que todos os grupos foram diferentes nas condições CV quando comparada a condição SV $(\mathrm{p}<0,05)$. Nesta condição, todos os grupos apresentaram uma maior velocidade de oscilaçáo na direção ML.

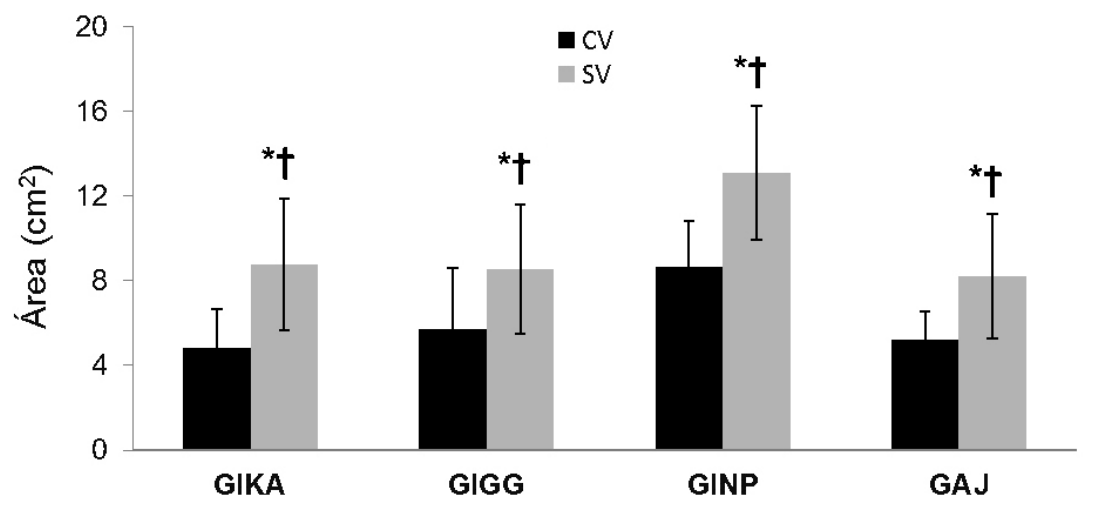

Média (Desvio-Padrão) dos valores de Área de oscilação do Cop em $\mathrm{cm}^{2}\left(^{*}=\right.$ diferença significativa entre as condições CV e SV $(p<0,05), \dagger=$ diferença significativa em relação ao GINP $(p<0,05))$.

FIGURA 1 - Área de oscilação.

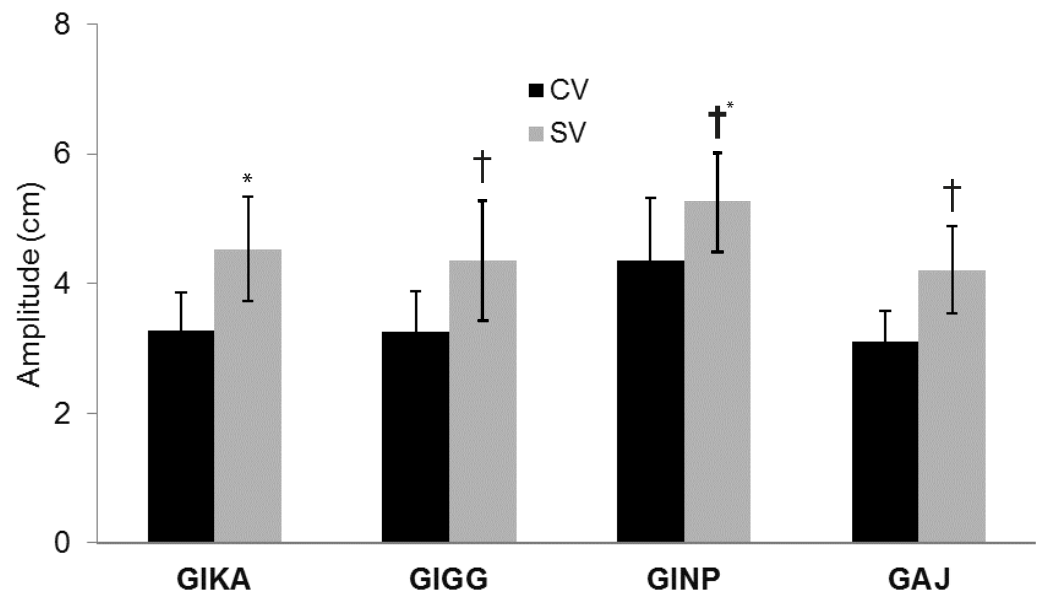

Média (DesvioPadrão) dos valores de Amplitude AP do Cop em $\mathrm{cm}\left({ }^{*}=\right.$ diferença significativa entre as condições CV e SV $(p<0,05), \dagger=$ diferença significativa em relação entre GINP e $\operatorname{GAJ}(p<0,05))$.

FIGURA 2 - Amplitude AP.

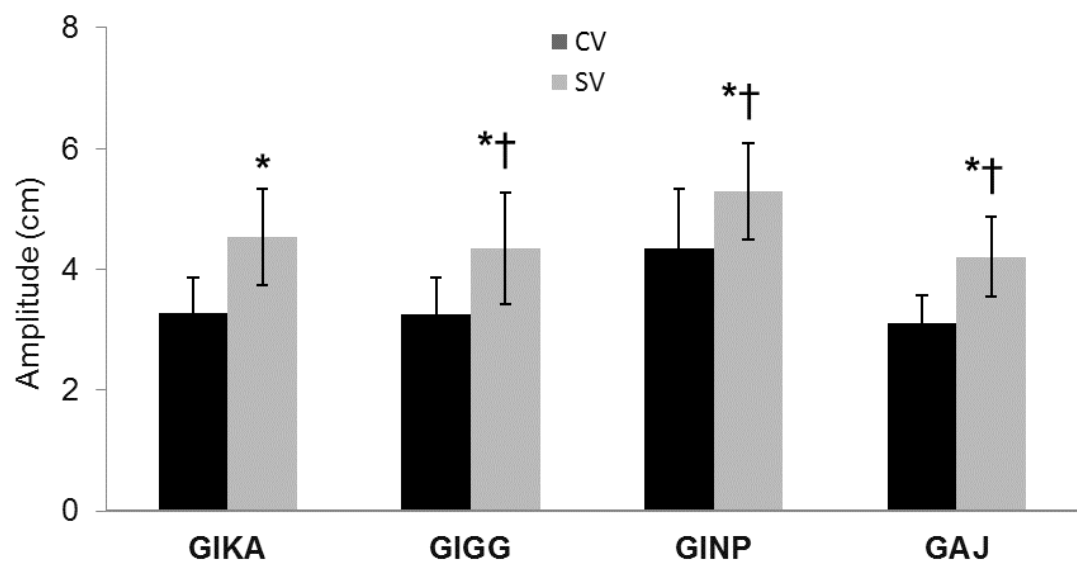

Média (Desvio-

Padrão) dos valores de Amplitude ML do Cop em $\mathrm{cm}\left({ }^{*}=\right.$ diferença significativa entre as condições CV e SV $(p<0,05), \dagger=$ diferença significativa em relação de GIGG E GINP e GINP e GAJ $(p<0,05))$.

FIGURA 3 - Amplitude ML. 
Média (DesvioPadrão) dos valores de Velocidade média de oscilação AP do Cop, em $\mathrm{cm} / \mathrm{s} ~^{*}=$ diferença significativa entre as condições CV e SV $(p<0,05), \dagger=$ diferença significativa entre os grupos GINP e GAJ $(p<0,05))$.
Média (DesvioPadrão) dos valores de Velocidade média de oscilação ML, do Cop, em $\mathrm{cm} / \mathrm{s}\left(^{*}=\right.$ diferença significativa entre as condições CV e SV $(p<0,05), \dagger=$ diferença significativa entre os grupos GIGG e GINP e GINP e GAJ $(p<0,05))$.

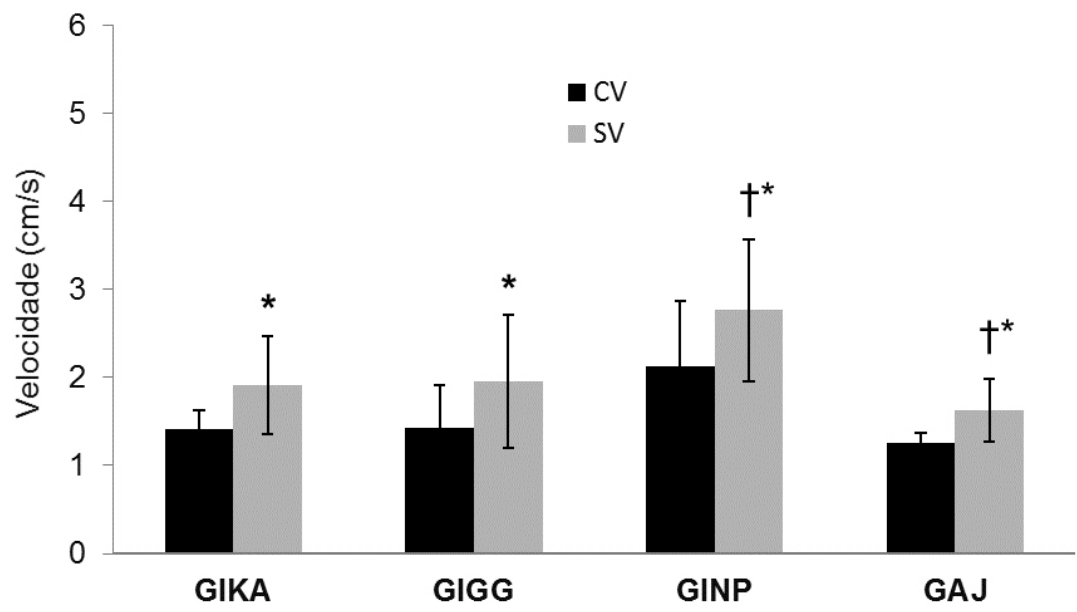

FIGURA 4 - Velocidade AP.

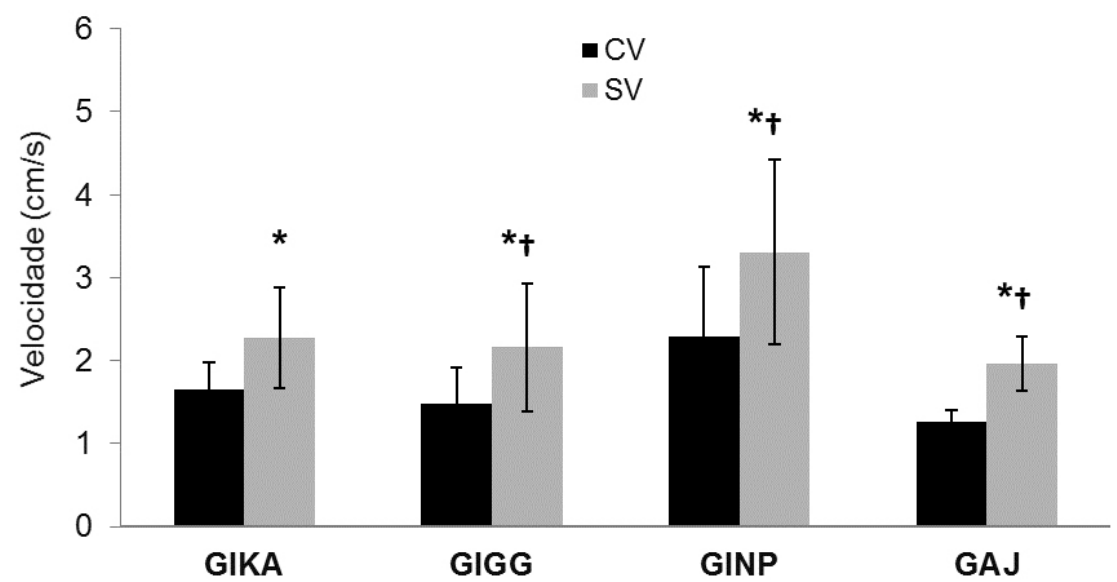

FIGURA 5 - Velocidade ML.

\section{Discussão}

O objetivo deste estudo foi comparar o equilíbrio postural de idosos praticantes de duas diferentes modalidades de exercícios físicos, idosos não praticantes de exercícios físicos e adultos jovens, com e sem informação visual.

$\mathrm{Na}$ condição sem informação visual todos os grupos foram mais instáveis no equilíbrio postural, sendo mais acentuado para o grupo de náo praticantes de exercícios físicos, ressaltando a influência da informaçáo sensorial da visão para o controle postural, sendo essa condição perturbadora para todos os grupos, inclusive ao de adultos jovens, corroborando os resultados de estudos que analisaram grupos de idosos ${ }^{30}$ e grupos de adultos ${ }^{31}$.
A hipótese do estudo não foi confirmada, no qual depreendeu-se que idosos praticantes de Karatê teriam um desempenho melhor, ou seja, com um menor deslocamento do Cop, nas variáveis analisadas, indicando um melhor equilíbrio postural quando comparado ao grupo de idosos praticantes de exercícios generalizados. Os grupos apresentaram resultados semelhantes, nas condiçóes com visão e sem visão.

De maneira geral, verificou-se que os idosos praticantes de exercícios físicos demonstraram menor oscilação postural, mais especificamente o grupo de idosos praticantes de ginástica generalizada, 
aproximando seus desempenhos ao de adultos jovens e diferindo-se de idosos não praticantes. Tais achados corroboram estudos prévios que compararam o equilíbrio postural de idosos praticantes de exercícios físicos e não praticantes ${ }^{6,25,32}$.

Alguns fatores podem ter sido responsáveis por esses resultados. Os grupos de idosos praticantes de exercícios físicos podem ter apresentado uma melhor integração sensório-motora, diminuindo a oscilação do centro de pressão na plataforma. Em estudo realizado por Prioli, Freitas e Barela ${ }^{25}$, verificouse que idosos ativos apresentaram deslocamento postural similar ao de adultos jovens. Resultados semelhantes também foram encontrados no estudo de Lamoth e van Heuvelen ${ }^{32}$, em que idosos praticantes de patinaçáo de velocidade no gelo foram comparados a idosos náo praticantes desta modalidade e adultos jovens. O equilíbrio postural dos idosos patinadores foi superior ao de idosos não patinadores e similares ao de adultos jovens, corroborando os resultados do presente estudo, em especial os achados referentes ao GIGG.

Esses resultados, de semelhanças no desempenho do controle postural entre idosos praticantes de exercícios físicos, corroboram com o estudo de Alfieri et al. ${ }^{14}$, assim como Gonzaga et al. ${ }^{16}$. Em outros delineamentos experimentais GAUCHARD et al..$^{33}$, no qual, verificaram o controle postural de idosos e Ueno et al. ${ }^{34}$ por meio da capacidade funcional de diferentes grupos, encontraram diferenças entre idosos praticantes de exercícios físicos, mostrando as características distintas das modalidades.

Dessa forma, observa-se que algumas combinações de modelos de programas de exercícios que buscam promover uma maior diversificação de movimentos e estímulos, por exemplo, treinamento de força associado a exercícios aeróbios, têm demonstrado maior eficiência no sentido de aprimorar os parâmetros funcionais e motores de idosos, tais como, coordenação, equilíbrio e flexibilidade ${ }^{35-37}$.
Alguns estudos já estão trazendo os benefícios de treinamentos mais específicos para a prevenção de quedas, como meio de minimizar a instabilidade postural e melhorar as reaçôes posturais em diferentes contextos $^{20,38}$. Com relação aos grupos terem sido semelhantes (GIGG e GIKA), a razão pode resultar das características das duas modalidades, visando o benefício do equilíbrio postural dos praticantes. Ambas enfatizam a conscientização corporal, priorizando a melhora das capacidades físicas e funcionais em seus treinamentos, corroborando com estudos prévios que não encontraram diferenças entre práticas de exercícios físicos no equilíbrio postural ${ }^{8,14}$.

Dessa forma, pode-se inferir, por meio dos resultados obtidos, independentemente do tipo de prática, haver uma melhora do equilíbrio postural. Consequentemente, os idosos praticantes de exercícios físicos, possivelmente, enfrentam menores riscos de instabilidades posturais e quedas. Compreende-se, dessa maneira, que existem diferenças sensóriomotoras características das especificidades das diversas práticas de exercícios físicos ou esportivas e que as mesmas influenciam o controle postural, mas no presente estudo as modalidades náo se diferenciaram, mostrando a importância da prática de exercícios físicos no envelhecimento, sendo que independente da modalidade, poderão ocorrer mudanças comportamentais importantes no equilíbrio postural.

Algumas limitaçóes do estudo podem ser atribuídas a esses resultados, como o número reduzido de participantes dos grupos. Outros estudos devem ser realizados com diferentes práticas de exercícios físicos nessa população, com número maior de participantes e com um maior controle relacionado aos exercícios praticados eàs frequências dos participantes.

Dessa forma, conclui-se que a prática do karatê e da ginástica generalizada para idosos pode levar a uma melhora do controle postural de maneira a aproximar ou igualar ao desempenho de adultos jovens.

\section{Abstract}

\section{Comparison of postural control in sedentary elderly, practitioners of different exercise modalities and young adults}

The aim of the study was to compare the postural balance of elderly practitioners of two exercise modalities (karate and gymnastics), sedentary elderly and young adults, with and without visual information. Fifty one individuals participated in this study, divided in four groups: elderly who practice karate (GIKA, $n=11)$, elderly practitioners of a general fitness program (GIGG, $n=16)$, sedentary elderly (GINP, $n=12$ ) and young adults 
(GAJ, $n=12$ ). The task consisted in remaining in static support (semi tandem position), on a force platform for 40 seconds. There were two experimental conditions: with and without vision. The posture control variables, obtained from the Center of Pressure (Cop) were: Cop's oscillation area, oscillation mean speed and oscillation mean amplitude, the last two both in the antero-posterior and medial-lateral directions. The results show that elderly in the GIKA and GIGG groups achieved similar performance, regardless of the exercise modality, both in the vision and no vision conditions. In the no vision condition, all groups were more susceptible to postural instability. The GINP group showed greater postural sway in all variables when compared to the other groups. The GIKA and GIGG groups had similar performance to GAJ. Thus, we conclude that the practice of physical exercise is effective in maintaining postural balance during the aging process.

\section{KeYwords: Aging; Postural balance; Physical exercise; Postural assessment.}

\section{Referências}

1. Brasil. Instituto Brasileiro de Geografia e Estatística. Censo Demográfico 2010: resultados gerais da amostra. Rio de Janeiro; 2010.

2. Marsh AP, Geel SE. The effect of age on the attentional demands of postural control. Gait Posture. 2000;12(2):105-13.

3. Weirich G, Bemben DA, Bemben MG. Predictors of balance in young, middle-aged, and late middle-aged women. Phys Ther. 2010;33(3):110-7.

4. Erickson KI, Kramer AF. Aerobic exercise effects on cognitive and neural plasticity in older adults. Br J Sports Med. 2008;43(1):22-4.

5. Brandalize D, Henrique P, Almeida F, Machado J, Endrigo R, Chodur A. Efeitos de diferentes programas de exercícios físicos na marcha de idosos saudáveis: uma revisão. Fisioter Mov. 2011;24(3):549-56.

6. Martins RM, Dascal JB, Marques I. Equilíbrio postural em idosos praticantes de hidroginástica e karatê. Rev Bras Geriatr e Gerontol. 2013;16(1):61-9.

7. Skelton DA. Effects of physical activity on postural stability. Age Ageing. 2001;30(Suppl 4):33-9.

8. Oliveira MR, Silva RA, Dascal JB, Teixeira DC. Effect of different types of exercise on postural balance in elderly women: a randomized controlled trial. Arch Gerontol Geriatr. 2014;59(3):506-14.

9. McPhee JS, French DP, Jackson D, Nazroo J, Pendleton N, Degens H. Physical activity in older age: perspectives for healthy ageing and frailty. Biogerontology. 2016;17(3):567-80.

10. Grabiner MD, Crenshaw JR, Hurt CP, Rosenblatt NJ, Troy KL. Exercise-based fall prevention: can you be a bit more specific? Exerc Sport Sci Rev. 2014;42(4):161-8.

11. Bressel E, Yonker JC, Kras J, Heath EM. Comparison of static and dynamic balance in female collegiate soccer, basketball, and gymnastics athletes. J Athl Train. 2007;42(1):42-6.

12. Agostini V, Chiaramello E, Canavese L, Bredariol C, Knaflitz M. Postural sway in volleyball players. Hum Mov Sci. 2013;32(3):445-56.

13. Hrysomallis C. Balance ability and athletic performance. Sports Med. 2011;41(3):221-32.

14. Alfieri FM, Riberto M, Gatz LS, Ribeiro CPC, Lopes JAF, Battistella LR. Comparison of multisensory and strength training for postural control in the elderly. Clin Interv Aging. 2012;7:119-25.

15. Alves RV, Mota J, Costa MC, Alves JGB. Aptidão física relacionada à saúde de idosos: influência da hidroginástica. Rev Bras Med Esp. 2004;10(1):31-7.

16. Gonzaga JDM, Barros SEB, Lisboa MGC, Barbieri FA, Gobbi LTB. Efeitos de diferentes tipos de exercício nos parâmetros do andar de idosas. Rev Bras Med Esporte. 2011;17(8):166-70.

17. Hang L, Yang F. Strength or power, which is more important to prevent slip-related falls? Hum Mov Sci. 2015;44:192-200.

18. Liu H, Frank A. Tai chi as a balance improvement exercise for older adults: a systematic review. J Geriatr Phys Ther. 2010;33(3):103-9.

19. Ni M, Mooney K, Richards L, Balachandran A, Sun M, Harriell K, et al. Comparative impacts of tai chi, balance training, and a specially-designed yoga program on balance in older fallers. Arch Phys Med Rehabil 2014;95(9):1620-8.e30.

20. Okubo Y, Schoene D, Lord SR. Step training improves reaction time, gait and balance and reduces falls in older people: a systematic review and meta-analysis. Br J Sports Med. 2016;51(7):583-93. 
21. Zago M, Mapelli A, Shirai YF, Ciprandi D, Lovecchio N, Galvani C, et al. Dynamic balance in elite karateka. J Electromyogr Kinesiol. 2015;25(6):894-900.

22. Pliske G, Emmermacher P, Weinbeer V, Witte K. Changes in dual-task performance after 5 months of karate and fitness training for older adults to enhance fall prevention. Aging Clin Exp Res. 2015;28(6):1179-86.

23. Vando S, Filingeri D, Maurino L, Chaabène H, Bianco A, Salernitano G, et al. Postural adaptations in preadolescent karate athletes due to a one week karate training cAMP. J Hum Kinet. 2013;38:45-52.

24. Witte K, Kropf S, Darius S, Emmermacher P, Böckelmann I. Comparing the effectiveness of karate and fitness training on cognitive functioning in older adults: a randomized controlled trial. J Sport Heal Sci. 2016;5(4):484-90.

25. Prioli AC, Freitas Júnior PB, Barela JA. Physical activity and postural control in the elderly: coupling between visual information and body sway. Gerontology. 2005;51(3):145-8.

26. Bonfim, TR R; Polastri, PF; Barela JA. Efeito do toque suave e da informação visual no controle da posição em pé de adultos. Rev Bras Educ Fís. 2006;20(1):15-25.

27. Perrot C, Mur JM, Mainard D, Barrault D, Perrin PP, Poincare H. Influence of trauma induced by judo practice on postural control. Scand J Med Sci Sports. 2000;10(5):292-7.

28. Gil AWO, Oliveira MR, Coelho V A, Carvalho CE, Teixeira DC, Silva RA. Relationship between force platform and two functional tests for measuring balance in the elderly. Rev Bras Fisioter. 2011;15(6):429-35.

29. Silva RA, Bilodeau M, Parreira RB, Teixeira DC, Amorim CF. Age-related differences in time-limit performance and force platform-based balance measures during one-leg stance. J Electromyogr Kinesiol. 2013;23(3):634-9.

30. Maki BE, McIlroy WE. Postural control in the older adult. Clin Geriatr Med. 1996;12(4):635-58.

31. Prado JM, Stoffregen TA, Duarte M. Postural sway during dual tasks in young and elderly adults. Gerontology. 2007;53(5):274-81.

32. Lamoth CJC, van Heuvelen MJG: Sports activities are reflected in the local stability and regularity of body sway: older iceskaters have better postural control than inactive elderly. Gait Posture. 2011;35:489-93.

33. Gauchard G, Jeandel C, Tessier A, Perrin P. Beneficial effect of proprioceptive physical activities on balance control in elderly human subjects. Neurosci Lett. 1999;273:81-4.

34. Ueno DT, Gobbi S, Teixeira CVL, Sebastião E, Prado AKG, Costa JLR, et al. Efeitos de três modalidades de atividade física na capacidade funcional de idosos. Rev Bras Educ Fís. 2012;26(2):273-81.

35. Cao Z-B, Maeda A, Shima N, Kurata H, Nishizono H. The effect of a 12-week combined exercise intervention program on physical performance and gait kinematics in community-dwelling elderly women. J Physiol Anthropol. 2007;26(3):325-32.

36. Lopopolo RB, Greco M, Sullivan D, Craik RL, Mangione KK. Research report effect of therapeutic exercise on gait speed in community-dwelling elderly people: a meta-analysis. Phys Ther. 2006;86:520-40.

37. Rubenstein LZ, Josephson KR, Trueblood PR, Loy S, Harker JO, Pietruszka FM, et al. Effects of a group exercise program on strength, mobility, and falls among fall-prone elderly men. J Gerontol A Biol Sci Med Sci. 2000;55(6):M317-21.

38. Segev-Jacubovski O, Herman T, Yogev-Seligmann G, Mirelman A, Giladi N, Hausdorff JM. The interplay between gait, falls and cognition: can cognitive therapy reduce fall risk? Expert Rev Neurother. 2011;11(7):1057-75.

\section{Agradecimentos}

Os autores são gratos pelo financiamento advindos da Fundação Araucária e do Conselho Nacional de Desenvolvimento Científico e Tecnológico (CNPq).

\begin{tabular}{|c|c|}
\hline ENDEREÇO & \\
\hline Raquel de Melo Martins & Recebido para publicação: 15/01/2015 \\
\hline Departamento de Educação Física & 1'a Revisão: 01/o6/2015 \\
\hline Universidade Estadual de Londrina & $2^{\text {a }}$ Revisão: 11/o8/2016 \\
\hline Rodovia Celso Garcia Cid, PR 445, $\mathrm{km} 380$ & 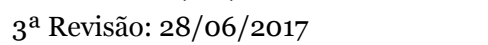 \\
\hline $\begin{array}{l}\text { 86020-430 - Londrina - PR - BRASIL } \\
\text { e-mail: quel_martins@hotmail.com }\end{array}$ & Aceito: 03/o8/2017 \\
\hline
\end{tabular}


